\title{
Design of Guidance Law for Extra-Atmospheric Vehicle Based on Eatimation of Maneuvering Target
}

\author{
Liu Peng ${ }^{*}$, Xue Feng, Liu Jiaqi \\ The National Key Lab of Computational Mathematics \& Experimental Physics, Beijing 100076, China \\ *Corresponding Author: lppl2008@163.com
}

\begin{abstract}
A modified augmented proportional navigation (MAPN) guidance law is proposed for the extra-atmospheric vehicle by using the adaptive extended Kalman filtering(AEKF) observer to estimate the target maneuver acceleration. The traditional Proportional Navigation (PN) guidance law has higher accuracy, in fact, it has been shown that PN is optimal solution for a stationary target. But for highly maneuvering targets, the accuracy of traditional PNG guidance law may sharply degrade. The Augmented Proportional Navigation (APN) guidance law add an acceleration compensation of the target which is introduced on the basis of PN guidance law to overcome the effect of the acceleration on the guidance accuracy. However, the target maneuver acceleration can not acquired directly with measurement, and a new method to estimate the target maneuver acceleration is established via the adaptive extended Kalman filtering(AEKF) observer in this paper. Mostly, APN law is powerful and useful for maneuvering target intercept, but the observability of the filter becomes depressed and divergent. In order to improve the observability of the system, the MAPNG law is derived by adding the line-of-sight (LOS) information into the APN. Simulation results indicated that, for homing against maneuverable targets, the MAPN guidance law is better than the PN guidance law in the following aspects : the higher guidance accuracy; the lower miss distance and the shorter interception time. It shows that the new guidance law provides significant performance improvements over the commonly used classical proportional navigation law.
\end{abstract}

Keywords: Extra-Atmospheric Vehicle, Modeling and Control, Maneuvering Target, AEKF, MAPN.

\section{Introduction}

Extra-Atmospheric Vehicle has an enormous, yet untapped potential as steady platform for monitoring and collision on missile-target, the research and exploitation of which have become a hot spot at home and abroad in recent years.

The PN guidance law has been analyzed very extensively in the literature ${ }^{(1)-(6)}$. Most guidance schemes in the literature are based on the principle of PN whose logic is to null the LOS rate by making missile heading rate proportional to the LOS (line of sight) rate while closing in on range ${ }^{(7)}$. Indeed, $\mathrm{PN}$ was shown to be optimal for linearized engagement equations in constant speed missile and static target intercept scenarios. However, its performance sharply degrades in the presence of rapidly maneuvering or fast moving targets.

In order to overcome miss distance problems in the presence of large target maneuvers, several modifications in the form of a bias added to PN for target acceleration compensation are suggested. One form of modification is the well known APN, where the commanded acceleration is a linear function of target acceleration as well ${ }^{(8)}$. But the target maneuver acceleration can not get directly, and is estimated via the AEKF observer. In order to improve the observability of the system, the MAPNG law is derived by adding the line-of-sight into the APN. Maneuvering target models are tested for performance comparison of the MAPN guidance with the PN guidance law.

\section{Extra-Atmospheric Vehicle Model}

\subsection{Propulsion System}

The extra-atmospheric vehicle is shown in figure 1 . The engine numberd in 5 10 is used for attitude control, and the $1 \sim 4$ is the orbit control engine. 


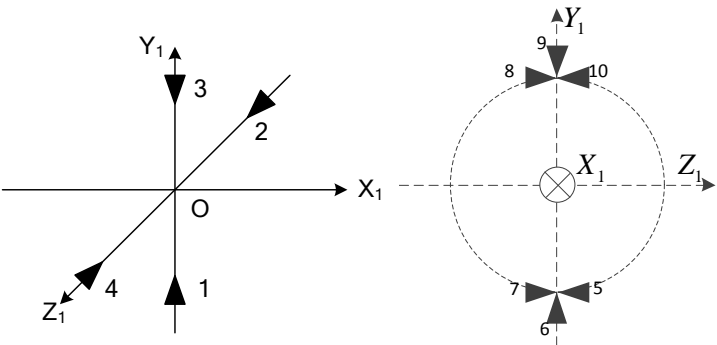

Fig. 1. Engine of attitude control and orbit control

\subsection{Kinematics Equations and Dynamic Equations}

The kinematics equations and dynamic equations is expressed in the inertial reference frame as follows,

$$
\begin{gathered}
m \frac{\delta^{2} r}{\delta t^{2}}=F_{c}+m g-m \omega_{e} \times\left(\omega_{e} \times r\right)-2 m \omega_{e} \times \frac{\delta r}{\delta t} \\
m \frac{\delta r}{\delta t}=V
\end{gathered}
$$

The rigid body rotated equation of the extra-atmospheric vehicle are :

$$
\left\{\begin{array}{l}
\dot{\omega}_{\mathrm{x}}=\frac{J_{\mathrm{y}}-J_{\mathrm{z}}}{J_{\mathrm{x}}} \omega_{\mathrm{y}} \omega_{\mathrm{z}}+\frac{M_{\mathrm{x} 1}}{J_{\mathrm{x}}} \\
\dot{\omega}_{\mathrm{y}}=\frac{J_{\mathrm{z}}-J_{\mathrm{x}}}{J_{\mathrm{y}}} \omega_{\mathrm{x}} \omega_{\mathrm{z}}+\frac{M_{\mathrm{y} 1}}{J_{\mathrm{y}}} \\
\dot{\omega}_{\mathrm{z}}=\frac{J_{\mathrm{x}}-J_{\mathrm{y}}}{J_{\mathrm{z}}} \omega_{\mathrm{x}} \omega_{\mathrm{y}}+\frac{M_{\mathrm{z} 1}}{J_{\mathrm{z}}}
\end{array}\right.
$$

The equations of motion are,

$$
\left\{\begin{array}{l}
\dot{\vartheta}=\omega_{\mathrm{y}} \sin \gamma+\omega_{\mathrm{z}} \cos \gamma \\
\dot{\psi}=\frac{1}{\cos \vartheta}\left(\omega_{\mathrm{y}} \cos \gamma-\omega_{\mathrm{z}} \sin \gamma\right) \\
\dot{\gamma}=\omega_{\mathrm{x}}-\tan \vartheta\left(\omega_{\mathrm{y}} \cos \gamma-\omega_{\mathrm{z}} \sin \gamma\right)
\end{array}\right.
$$

\section{Estimation of the Maneuvering Target Acceleration}

When comes to the terminal guidance, there only has the line-of-sight angle information provided by the infrared seeker, and the information of target maneuver acceleration used in guidance law was estimated via the observer by using the angle information provided by the infrared seeker.

The AEKF algorithm was used in estimating the maneuvering target acceleration, and the AEKF algorithm are given by equations from(5) to (10).

$$
\mathbf{x}(k / k)=\mathbf{x}(k / k-1)+\mathbf{K}(k) \mathbf{\varepsilon}(k)
$$

$$
\begin{gathered}
\boldsymbol{\varepsilon}(k)=\mathbf{z}(k)-\mathbf{H}(k) \mathbf{x}(k / k-1)-\mathbf{r}_{1} \\
\mathbf{x}(k / k-1)=\mathbf{\Phi}(k-1) \mathbf{x}(k-1 / k-1)+\boldsymbol{\Gamma}(k-1) \mathbf{u}(k-1)+\mathbf{q}_{1} \\
\mathbf{K}(k)=\mathbf{P}(k / k-1) \mathbf{H}^{T}(k)\left[\mathbf{H}(k) \mathbf{P}(k / k-1) \mathbf{H}^{T}(k)+\mathbf{R}_{1}\right]^{-1} \\
\mathbf{P}(k / k-1)=\mathbf{\Phi}(k-1) \mathbf{P}(k-1 / k-1) \mathbf{\Phi}^{T}(k-1)+\mathbf{Q}_{1} \\
\mathbf{P}(k / k)=[\mathbf{I}-\mathbf{K}(k) \mathbf{H}(k)] \mathbf{P}(k / k-1)
\end{gathered}
$$

In the observer design, the target maneuver was viewed as external disturbance input, so it was not necessary to construct a target maneuver model in the design of the observer, and no statistical assumption was made on target maneuver. By introducing the adaptive fading factor, the AEKF algorithm limits the memory length and full uses the current measurement data, greatly enhance the filtering tracking performance, and it has better robustness than the traditional KF algorithm ${ }^{(9)}$.
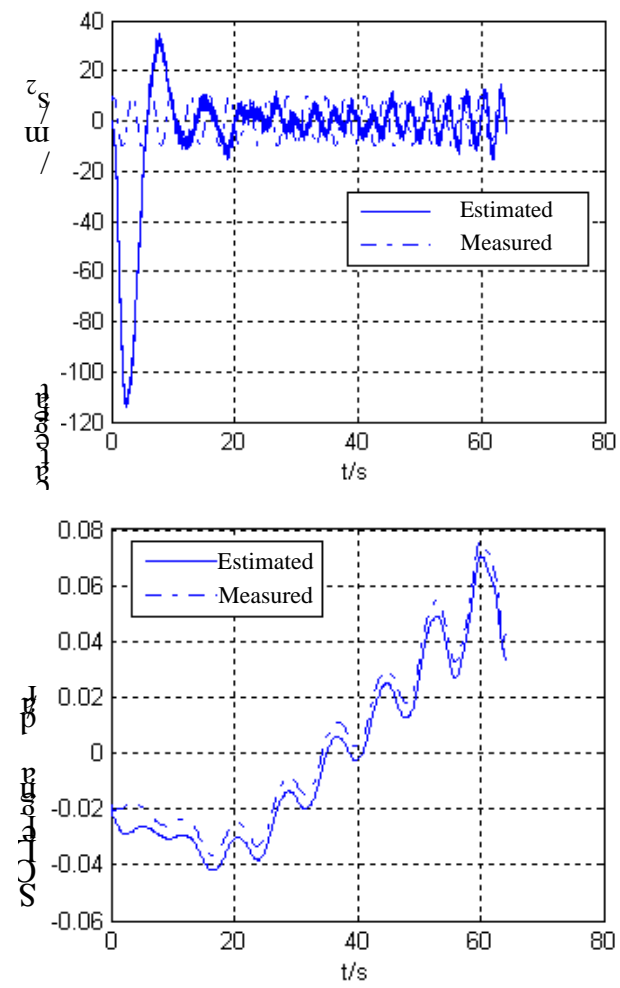

Fig 2: Estimation of the maneuvering target acceleration results

Simulation results in Fig 2 shows that the AEKF has good performance in estimating the maneuvering target acceleration.

\section{Control System Design}

The control system consist of attitude control system 
and orbit control system, the attitude control system used PID controller and the orbit control system used augmented proportional navigation method. The conrol logic is designed for attitude control engine and orbit control engine with fixed thrust. The control system frame is shown in figure 3.

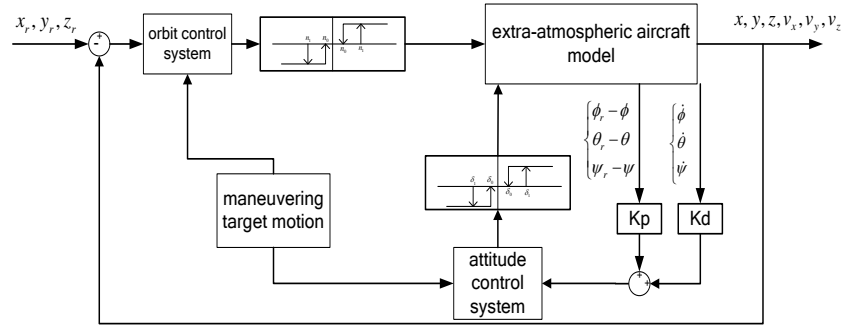

Fig 3. The control system frame of the extra-atmospheric vehicle

\subsection{Attitude control System Design}

\subsubsection{Attitude Control Law}

The attitude control law of the longitude channel is expressed as follows,

$$
\delta_{\text {lon }}=k_{p}\left(\theta_{r}-\theta\right)+k_{d}\left(\dot{\theta}_{r}-\dot{\theta}\right)
$$

The threshold of the attitude control engine is designed as :

$$
\left\{\begin{array}{l}
\delta_{0}=k_{01}\left(\frac{F_{z \max }\left(\tau+t_{0}\right) T L_{x}}{2 J_{z}}\right. \\
\delta_{1}=k_{11}\left(\frac{F_{z \max }\left(\tau+t_{0}\right) n T L_{x}}{2 J_{z}}\right)
\end{array}\right.
$$

If the requied engine conrol input is bigger than the threshold, $|\delta|>\left|\delta_{1}\right|$, the attitude control engine is working, $M_{z}=-F_{z} L_{x} \operatorname{sgn}(\delta)$, until the control input is lower than the threshold, $|\delta|<\left|\delta_{0}\right|$, the attitude control engine is closed, $M_{z}=0$.

In the same way, we can get the control law of the roll and the yaw channel

$$
\begin{aligned}
& \delta_{\text {lat }}=k_{p}\left(\gamma_{r}-\gamma\right)+k_{d}\left(\dot{\gamma}_{r}-\dot{\gamma}\right) \\
& \delta_{\text {ped }}=k_{p}\left(\psi_{r}-\psi\right)+k_{d}\left(\dot{\psi}_{r}-\dot{\psi}\right)
\end{aligned}
$$

\subsubsection{Simulation}

Noting that there consists coupling between different channels, the control inputs are added in three channels in the same time (pitch angle $15^{\circ}$, yaw angle $10^{\circ}$ and roll angle $5^{\circ}$ ) , and the step responses are shown in figure 4 :
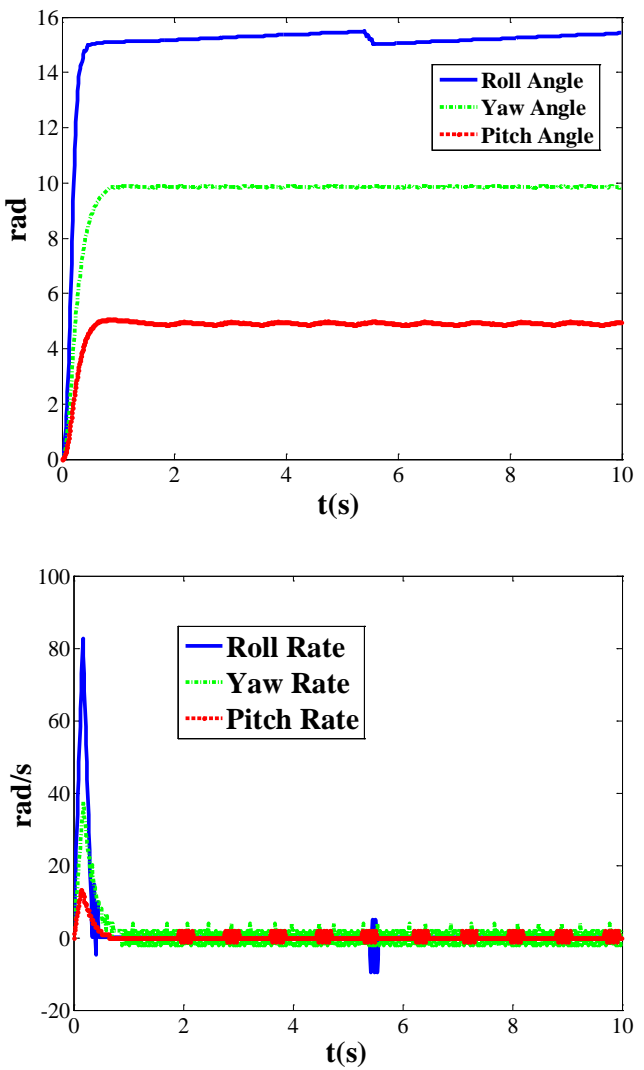

Fig 4. The step response of three channels

\subsection{Orbit Control System Design}

\subsubsection{Target Motion Equation and the PN Method}

The equations of the target motion towards the extra-atmospheric vehicle is expressed in the target- line of sight frame(4) :

$$
\left\{\begin{array}{l}
\ddot{R}-R\left(\omega_{y s}^{2}+\omega_{z s}^{2}\right)=-a_{x s} \\
R \dot{\omega}_{z s}+2 \dot{R} \omega_{z s}=-a_{y s} \\
R \dot{\omega}_{y s}+2 \dot{R} \omega_{y s}=a_{z s}
\end{array}\right.
$$

The structure of the standard PN is given by,

$$
a_{\mathrm{m}_{\beta}}=N V_{\mathrm{c}} \dot{\beta}
$$

where, $\mathrm{N}$ is the navigation constant.

\subsubsection{Engine Control with the Fixed Thrust}

The control law of the orbit control engine is 
designed based on the targer motion equation as follows,

$$
F_{y}=-m \cdot N \frac{\omega_{z s}(0)}{N-1} \frac{R_{0}}{T_{c}}\left(\left(1+\frac{\dot{R}}{R_{0}} T_{c}\right)^{N-1}-1\right)
$$

If the requied engine conrol input $\mathrm{F}_{\mathrm{y}}$ is bigger than the fixed thrust $\mathrm{P}_{0}, F_{\mathrm{y}}>P_{0}$, the orbit control engine is working, until the conrol input is lower than the fixed thrust, $F_{\mathrm{y}}<P_{0}$, the orbit control engine is closed, $F_{y}=0$.

In the same way, we can get the control law of the other channel:

$$
F_{z}=-m \cdot N \frac{\omega_{y s}(0)}{N-1} \frac{R_{0}}{T_{c}}\left(\left(1+\frac{\dot{R}}{R_{0}} T_{c}\right)^{N-1}-1\right)
$$

\subsubsection{MAPN Method}

The traditional PN guidance law has higher accuracy. But for highly maneuvering targets, the accuracy of traditional PN guidance law is still not enough. An APN guidance law is designed, an acceleration compensation of the target is introduced on the basis of PN guidance law to overcome the effect of the acceleration on the guidance accuracy. The structure of the APN is given by ${ }^{(10)}$ :

$$
a_{\mathrm{m}_{\beta}}=N V_{\mathrm{c}} \dot{\beta}+\frac{N}{2} a_{\mathrm{t}_{\beta}}
$$

where, $\mathrm{N}$ is the navigation constant and $a_{\mathrm{t}_{\beta}}$ is the target acceleration information.

APN law is power and useful for maneuvering target, but it makes $\dot{\beta} \rightarrow 0$, the observability of the system becomes depressed, and the filter used to estimate the maneuvering target acceleration will be unconverged.

In order to improve the observability of the system, we derived the MAPNG law by adding the $k R \beta$ into the APN,

$$
a_{\mathrm{m}_{\beta}}=N V_{\mathrm{c}} \dot{\beta}+\frac{N}{2} a_{\mathrm{t}_{\beta}}+F R \beta
$$

As,

$$
R \ddot{\beta}-2 V_{\mathrm{c}} \dot{\beta}=a_{\mathrm{t}_{\beta}}-a_{\mathrm{m}_{\beta}}
$$

Substituting the equation(15) into(14) yields,

$$
R \ddot{\beta}-2 V_{\mathrm{c}} \dot{\beta}=a_{\mathrm{t}_{\beta}}-\left(N V_{\mathrm{c}} \dot{\beta}+\frac{N}{2} a_{\mathrm{t}_{\beta}}+F R \beta\right)
$$

$\mathrm{R}$ can be represented by $R=V_{\mathrm{c}}\left(t_{\mathrm{f}}-t\right)$, and then we can get the following equation,

$$
\ddot{\beta}+\frac{N-2}{t_{\mathrm{f}}-t} \dot{\beta}+F \beta=\frac{2-N}{2 V_{\mathrm{c}}\left(t_{\mathrm{f}}-t\right)} a_{\mathrm{t}_{\beta}}
$$

From equation (17) we can see that the $\beta$ and $\dot{\beta}$ will be oscillated in the terminal guidance, and the observability of the system is obviously improved. When $t \rightarrow t_{\mathrm{f}}$, $k R \beta \rightarrow 0$, and the performance of the APNG and MAPNG law becomes similar.

\subsubsection{Simulation Resul}

The simulations are run for both using the PN method and APN method. The guidance methods are compared for two methods. Miss distance and simulation duration performances of the methods are given in Table 1 as follows, and the simulations results are shown in Figure5 6.

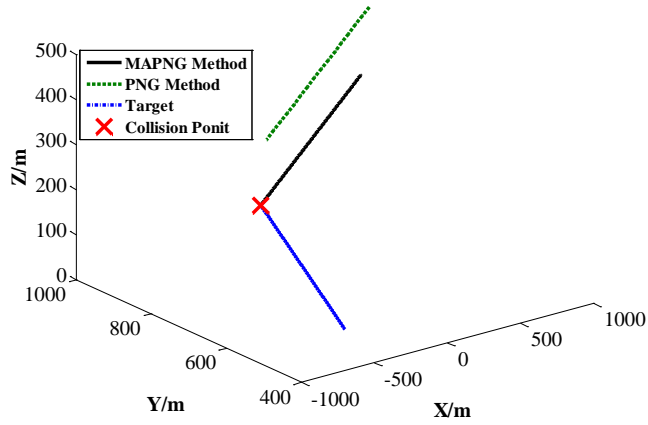

(a) The trajectory of the interception

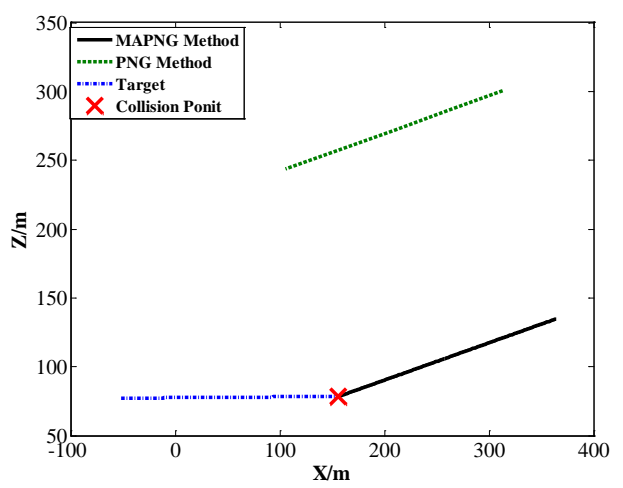

(b) XOZ plane 


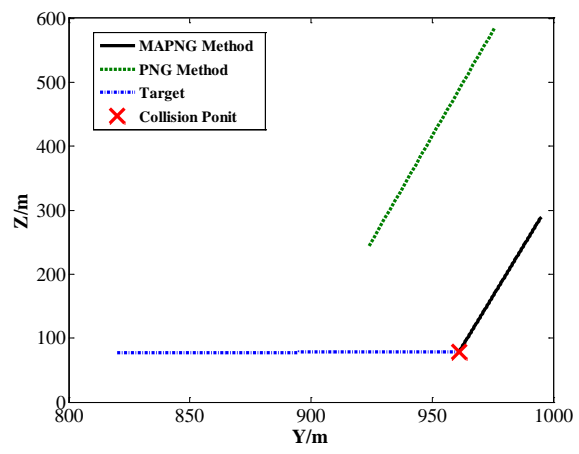

(c) YOZ plane

Fig 5. The simulation results with two guidance law
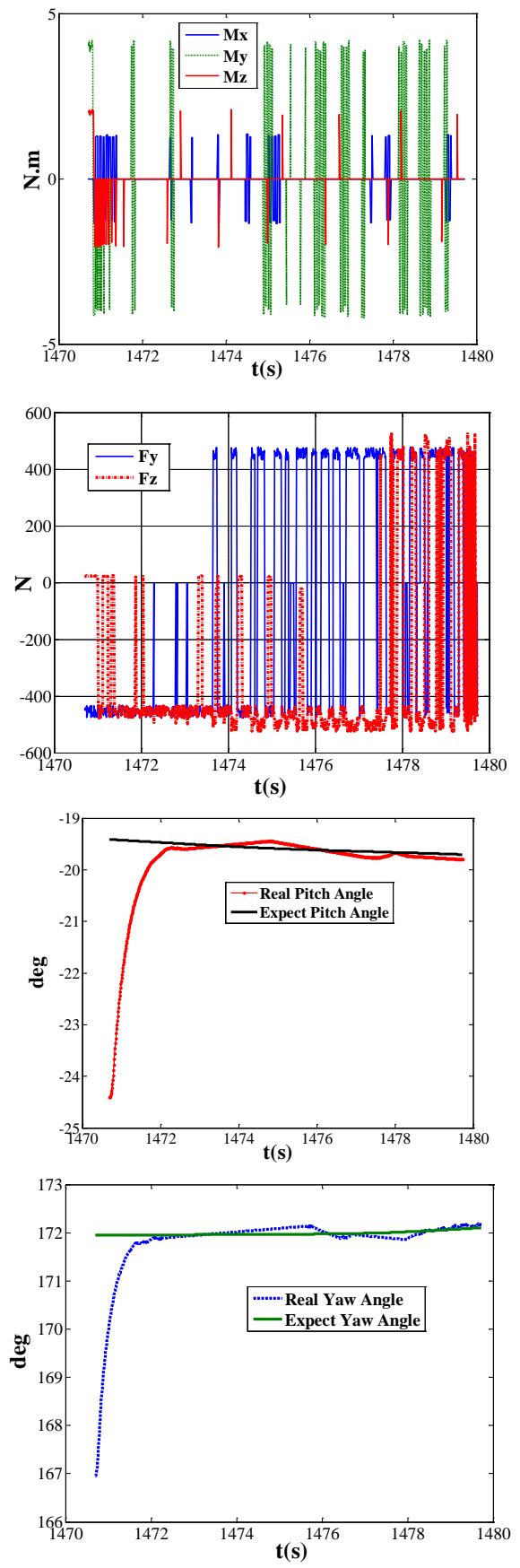

Fig 6. The result of the simulation
According to the results obtained, for homing against maneuverable targets, the MAPN guidance law is better than the PN guidance law in the following aspects : guidance accuracy is higher, miss distance is lower, interception time is shorter. And the new guidance law provides significant performance improvements over the commonly used classical proportional navigation law.

Table 1. Comparison of PN and MAPN guidance law

\begin{tabular}{|c|c|c|c|}
\hline Performance & $\begin{array}{c}\text { Fuel } \\
\text { Consume (kg) }\end{array}$ & $\begin{array}{c}\text { Inerception } \\
\text { time (s) }\end{array}$ & $\begin{array}{c}\text { Miss distance } \\
(\mathrm{m})\end{array}$ \\
\hline PNG & 2.97 & 8.87 & 180.1 \\
\hline MAPNG & 2.68 & 8.75 & 0.0024 \\
\hline
\end{tabular}

\section{Conclusions}

A new method for missile guidance law is suggested in this work to improve the effectiveness of PN guidance law against fast maneuvering targets. The extra-atmospheric vehicle model is established, and then the control system including attitude control system and orbit control system are designed.

For highly maneuvering targets, the APN guidance law is powerful, an important advantage of APN guidance methodology is that an acceleration compensation of the target is introduced on the basis of PN guidance law to overcome the effect of the acceleration on the guidance accuracy, but the target maneuver acceleration can not get directly, and is estimated via the AEKF observer of which the observability becomes depressed when use the APN method. In order to improve the observability of the system, the MAPN guidance law is derived by adding the LOS angle information into the APN guidance law.

Simulation results shows that for maneuvering vehicle interception, proposed new guidance formulation have improved performance outputs than PN guidance law.

\section{References}

(1) C.L.Yuan,"Homing and navigational courses of automatic target-seeking devices”,J.Applied Physics,19,pp.1122-1128,Dec 1988.

(2) S.A.Murtaugh and H.E.Criel,"Fundamentals of proportional navi-gation”, IEEE Spectrum,3(6),pp.75—85,Dec 1996.

(3) M.Guelman,"A qualititive study of proportional 
navigation",

IEEE

Trans.on

AES,AES-7(4),pp.637—643,July 1991.

(4) K.Becker,"Closed form solution of pure proportional navigation",

IEEE

Trans.on

AES,AES-26(3),pp.526-533,May 2000.

(5) M.Guelman,"The closed-form solution of true proportional naviga-tion”, IEEE Trans.on AES,AES-12(4),pp.472—482,July 2006.

(6) P.J.Yuan and J.S.Chern,"Solutions of true proportional navigation for maneuvering and non-maneuvering targets”, J.Guidance,Control, and Dynamics,15(1),pp.268-271,Jan-Feb 2002.

(7) Babu,K.,R.,Sarma,I.,G.and Swamy,K.,N.,"Switched Bias Proportional Navigation for Homing Guidance against Highly Maneuvering Targets”,Journal of Guidance,Control and Dynamics,vol.17,no.6,p:1357-1363,November-Decem ber 2008

(8) Zarchan,P.,"Tactical and Strategic Missile Guidance",American Institute of Aeronautics and Astronautics Inc.,2004.

(9) Greg Welch, Gary Bishop. An Introduction to the Kalman Filter. UNC-Chapel Hill, TR95-041, July 24. 2006

(10) Fumiaki Imado,Makoto Nagayama,Min-jea Tahk,An Extended Study on A New Missile Guidance Algorithm Against A maneuvering Target, AIAA Guidance, Navigation, and Control Conference and Exhibit,8.2001. 